\title{
Sunitinib inhibits tumor vascularity and growth but does not affect Akt and ERK phosphorylation in xenograft tumors
}

\author{
PASQUALE VOCE ${ }^{1}$, MARIA D'AGOSTINO $^{2}$, SONIA MORETTI $^{1}$, MARIALUISA SPONZIELLO $^{3}$, \\ KERRY RHODEN $^{4,5}$, FILIPPO CALCINARO ${ }^{1}$, GIULIA TAMBURRANO ${ }^{3}$, GIOVANNI TALLINI ${ }^{4,5}$, \\ EFISIO PUXEDDU ${ }^{1}$, SEBASTIANO FILETTI $^{3}$, DIEGO RUSSO $^{2}$ and COSIMO DURANTE ${ }^{3}$

\begin{abstract}
${ }^{1}$ Department of Internal Medicine, University of Perugia, I-06126 Perugia; ${ }^{2}$ Department of Pharmacobiological Sciences, University of Catanzaro 'Magna Graecia', I-88100 Catanzaro; ${ }^{3}$ Department of Internal Medicine and Medical Specialties, University of Rome 'Sapienza', I-00161 Rome; ${ }^{4}$ Department of Hematology and Oncological Sciences 'L. e A. Seragnoli' and ${ }^{5}$ Department of Gynaecological, Obstetric and Pediatric Sciences, University of Bologna, I-40139 Bologna, Italy
\end{abstract}

Received April 4, 2011; Accepted May 5, 2011

DOI: 10.3892/or.2011.1422

\begin{abstract}
Sunitinib is a multikinase inhibitor approved for use in some human solid malignancies, including renal clear cell and gastrointestinal stromal cancer, and under investigation for many other neoplasias. In many preclinical cancer models sunitinib has shown anti-angiogenic and antitumor effects, acting mainly by inhibiting the activity of pro-angiogenic growth factor receptors. However, a percentage of tumors develop resistance to this treatment. The aim of this study was to identify novel potential molecular targets for the nonresponsive tumors. The effects of sunitinib were investigated in xenograft tumors obtained by injecting HEK293 cells into NOD-SCID mice, focusing on the activity of growth-regulating pathways involved in tumorigenesis. During 11 days of oral administration of sunitinib ( $40 \mathrm{mg} / \mathrm{kg} / \mathrm{day})$, the growth of tumors was monitored by measuring the mass volume by a caliper. At the end of the treatment, tumor specimens were histologically examined for microvessel density (MVD) and presence of necrosis, and the phosphorylation of ERK and Akt was analyzed in protein extracts by Western blotting. Moreover, the mRNA levels of VEGF and its receptor genes were measured by quantitative RT-PCR. Treatment with sunitinib elicited a clear reduction of the tumor growth, associated with a reduction of MVD, correlated with an increased number of necrotic cells. In contrast, the levels of phosphorylated Akt and ERK proteins were similar in treated and non-treated animals. The VEGF and VEGFR-1 and 2 transcripts were not affected by sunitinib treatment. In conclusion, these findings
\end{abstract}

Correspondence to: Dr Diego Russo, Department of Pharmacobiological Sciences, University of Catanzaro 'Magna Graecia', Campus Universitario, Germaneto, Viale Europa, I-88100 Catanzaro, Italy E-mail: d.russo@unicz.it

Key words: sunitinib, Akt, ERK, vascular endothelial growth factor confirm the anti-angiogenic action as the major effect of sunitinib against tumor growth. In contrast, other important growth regulatory pathways involved in malignant transformation, such as the ERK-MAPK and Akt/mTOR pathways are not affected by such a treatment, suggesting the use of specific inhibitors of these pathways as valid candidates for combinatorial therapies in sunitinib-resistant malignancies.

\section{Introduction}

Sunitinib (sunitinib malate; SU11248; Sutent ${ }^{\circledR}$, Pfizer Inc., New York, NY, USA) is a multitargeted tyrosine kinase (TK) inhibitor with antitumor and anti-angiogenic activities. Sunitinib has shown potent inhibiting effects on the activity of vascular endothelial growth factor (VEGF) receptors one (VEGFR-1) and two (VEGFR-2), platelet-derived growth factor receptor (PDGFR), fetal liver TK receptor and KIT (stem-cell factor receptor), in both biochemical and cellular assays $(1,2)$. Moreover, many preclinical and clinical studies support its efficacy in renal cancer and gastrointestinal stromal tumors (GIST), showing as its major mechanism of action inhibition of the activity of VEGFR and PDGFR (3). In various tumors sunitinib has also displayed antiproliferative and apoptotic effects via inhibiting the protein TK-mediated proliferation and survival of tumor cells (4).

Although sunitinib is currently considered the standard of care for first-line therapy of metastatic clear-cell renal cell carcinoma (RCC) (5), not all patients respond to this drug and the vast majority eventually develop resistance to this therapy (6). Similarly, in GIST resistant to imatinib, the treatment with sunitinib may also result in a secondary TK inhibitor resistance (7). Up-regulation of alternative kinase pathway has been proposed as one of the mechanisms of resistance to kinase inhibitors developing in tumor cells $(8,9)$. Thus, in this preclinical study we investigated the in vivo effects of sunitinib on the activity of some tumorigenic pathways. In xenograft tumors obtained by injecting HEK293 cells in immunodeficient mice, the levels of phosphorylated ERK and Akt were analyzed after treatment with sunitinib (40 $\mathrm{mg} / \mathrm{kg}$ /day for 11 days), able 
to inhibit tumor growth by reducing its vascularity. In addition, the gene expression of the proangiogenic factor VEGF and its receptors was also evaluated.

\section{Materials and methods}

Xenograft model. Animal experiments were carried out in agreement with the principles and procedures approved by the local Ethics Committee. HEK293 cells were cultured in DMEM (Lonza, Milan, Italy) containing 10\% fetal bovine serum, penicillin $(0.1 \mathrm{mg} / \mathrm{ml})$ and streptomycin $(2.5 \mu \mathrm{g} / \mathrm{ml})$ (SigmaAldrich S.r.l., Milan, Italy). Female NOD-SCID mice of 4-6 weeks of age were injected subcutaneously in the flank with $1 \times 10^{6}$ cells suspended in $200 \mu \mathrm{l}$ of D-PBS. Treatment was initiated when tumors reached an average volume of $\sim 500 \mathrm{~mm}^{3}$. Tumor-bearing mice were randomized into groups ( $\mathrm{n}=5$ each) consisting of a control group (vehicle only) and treatment group with sunitinib $(40 \mathrm{mg} / \mathrm{kg} / \mathrm{day}$ for 11 days). Treatment was delivered once daily by oral gavage in a volume of $\sim 100 \mu 1$ using a sterile animal feeding needle. Tumor volume was measured at the start of the treatment and every 2 days during the course of therapy using a caliper, and tumor volumes calculated according to the following equation: $\mathrm{Vt}=0.5 \mathrm{x} \mathrm{Dd}^{2}$ where $\mathrm{Vt}$ is the volume of tumor, $\mathrm{D}$ and $\mathrm{d}$ the long and short diameter. The body weight, feeding behaviour and motor activity of mice were used as indicators of general health. At sacrifice, the tumors were dissected free of vessels, fibrous tissue and surrounding dermis. Tumors were weighed, cut longitudinally to provide a representative fragment for immunohistochemistry, and the remainder was fresh frozen in liquid $\mathrm{N}_{2}$ for subsequent protein and RNA isolation.

Protein extraction and Western blot analysis. Total proteins were extracted from xenograft tumor tissue and homogenized in $150 \mu \mathrm{l}$ of buffer containing $\mathrm{NaCl} 0.15 \mathrm{mM} / 1, \mathrm{MgCl}_{2} 1.5 \mathrm{mM} / 1$, HEPES $50 \mathrm{mM} / 1$, EGTA $5 \mathrm{mM} / 1$, glycerol $1 \% \mathrm{v} / \mathrm{v}$, Triton $1 \%$ $\mathrm{v} / \mathrm{v}$ (all from Sigma-Aldrich) and complete protease inhibitor cocktail (Roche Diagnostics, Milan, Italy). The homogenate was centrifuged at $1200 \mathrm{rpm}\left(4^{\circ} \mathrm{C}\right.$ for $\left.10 \mathrm{~min}\right)$, the supernatant containing the whole-cell lysate was centrifuged at $12000 \mathrm{rpm}$ $\left(4^{\circ} \mathrm{C}\right.$ for $\left.15 \mathrm{~min}\right)$ and were quantified spectrophotometrically using the Bradford method. Thirty micrograms of proteins were loaded onto a $10 \%$ SDS-polyacrylamide gel and subjected to electrophoresis at a constant voltage $(120 \mathrm{~V})$. Electroblotting to a Hybond ECL-PVDF membrane (GE Healthcare, Milan, Italy) was performed for $2 \mathrm{~h}$ at $225 \mathrm{~mA}$ using the Mini Trans Blot system (Bio-Rad Laboratories, Milan, Italy). Membranes were blocked with TTBS/milk (TBS, $1 \% \mathrm{v} / \mathrm{v}$ Tween-20, and 5\% $\mathrm{w} / \mathrm{v}$ non-fat dry milk) for $1 \mathrm{~h}$ at room temperature and incubated with affinity-purified anti-Akt, anti-p-Akt polyclonal antibody diluted 1:200 (Santa Cruz Biotechnology, Santa Cruz, CA, USA), anti-ERK1,2 and anti-p-ERK1,2 polyclonal antibody diluted 1:1000 (Cell Signaling Technology, Danvers, MA, USA). After one 15-min and two 5-min washes in TTBS, the membrane was incubated with horseradish peroxidaseconjugated secondary antibodies (Sigma-Aldrich) (1:5000) in TTBS/milk. After one 15-min and two 5-min washes in TTBS, the protein was visualized with an enhanced chemiluminescence Western blot analysis detection system (ECL plus, GE Healthcare).
RNA isolation, reverse transcription and quantitative realtime PCR analysis. Transcripts levels of VEGF-A and VEGFR1, 2 and 3 genes were determined using real-time quantitative RT-PCR as previously described (10). Briefly, $1 \mu \mathrm{g}$ of totalRNA, extracted from tumor tissues using Trizol method (Invitrogen, Carlsbad, CA, USA), was reverse transcribed in a $20 \mu 1$ reaction volume using the High Capacity cDNA Reverse Transcription kit (Applied Biosystems, Foster City, CA, USA) according to the manufacturer's instructions. The complementary DNAs (cDNAs) were then diluted 1:5 in nuclease-free $\mathrm{H}_{2} \mathrm{O}$ (Gibco, Milan, Italy) and amplified in an Applied Biosystems 7900HT fast real-time PCR Sequence Detection System (Applied Biosystems) using fast quantitative PCR thermal cycler parameters. Each tube (reaction mixture volume: $20 \mu \mathrm{l}$ ) contained $2.0 \mu \mathrm{l}$ of cDNA, $10.0 \mu \mathrm{l}$ TaqMan Fast Universal PCR master mix (Applied Biosystems), $1.0 \mu \mathrm{l}$ of a primer/probe mixture for each of the genes evaluated (VIC dye-labelled probes, Assay-on-Demand Gene Expression Products, Applied Biosystems). The $\beta$-actin gene was used as an endogenous reference (Pre-Developed TaqMan Assay Reagents; VIC dye-labeled probes P/N 4326315E; Applied Biosystems). All amplification reactions were performed in triplicate; the threshold cycle $\left(\mathrm{C}_{t}\right)$ was obtained using Applied Biosystem software (SDS version 2.3) and was averaged. Results were determined by the $2^{-\Delta \Delta \mathrm{Ct}}$ method and were expressed as relative expression normalized to a calibrator (control mice).

Assessment of tumor necrosis and of microvessel density (MVD). Specimen of xenografted tumors from three sunitinib-treated mice and three non-treated controls were fixed in formalin and embedded in paraffin for routine histologic examination after hematoxylin and eosin staining. The amount of tumor necrosis and MVD were assessed microscopically on 10 whole tumor sections from three sunitinib-treated mice (3 whole sections for each tumor) and on 8 whole tumor sections from the three controls. Tumor necrosis was assessed after complete scanning of whole tumor sections at $\times 100$ as the percentage of each whole tumor section showing areas of breakdown with eosinophilic cellular debris, nuclear disruption and degenerative changes. Microvessel density was evaluated after immunostaining with anti-CD31 antibodies (Cell Marque, Rocklin, CA, USA) following the Weidner protocol (11) with some modifications. Briefly, by scanning each whole tumor section at low power (x40 and x100, magnification) tissue vascularity was graded from 1 to 3 , to determine vascular hot spots. Then, four areas with the highest vessel density were selected for each whole tumor section, individual vessels were counted at high power (x200), and the average values of the four readings were recorded for statistical analysis.

Statistical analysis. Results of growth analysis are expressed as means $\pm \mathrm{SD}$ and differences were analyzed with the t-test. P-value $<0.05$ was used as the cut off for statistical significance. Quantitative RT-PCR results are expressed as means \pm SD and differences were analyzed with the t-test. P-value $<0.05$ was used as the cut-off for statistical significance. Data analysis was performed using StatView 5.0.1 software (SAS Institute, Inc., Cary, NC, USA). The percentage of tumor necrosis and the average MVD readings for sunitinib-treated and control 
A

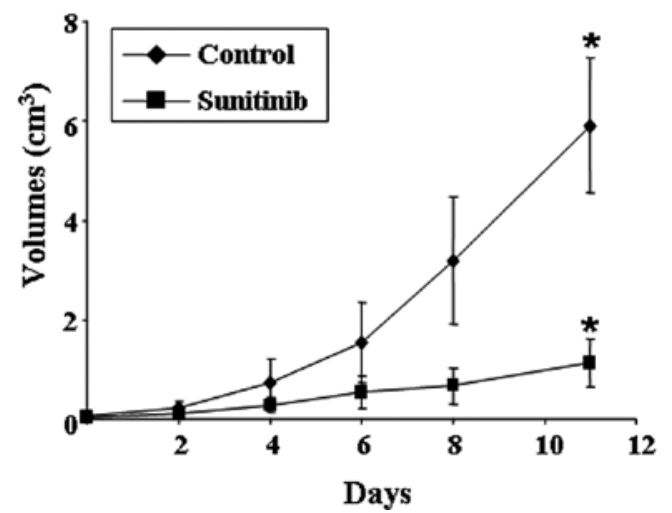

B

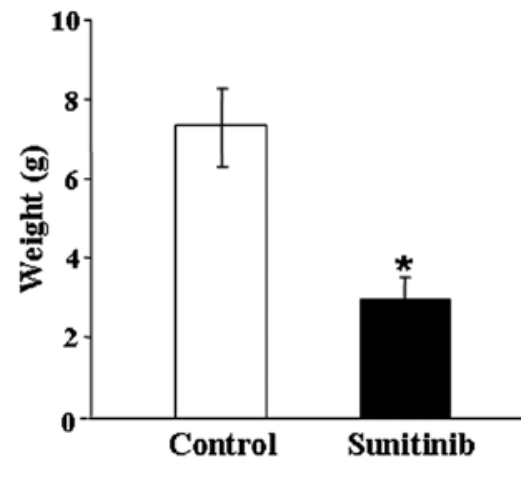

Figure 1. In vivo antitumoral effects of Sunitinib (40 mg/kg/day) in immunodeficient NOD-SCID mice bearing xenograft tumors. (A) Variation of tumor volume as a function of duration of treatment. (B) At the end of the experiments, animals were sacrificed and the tumor masses were excised and weighed. Animals were treated for 11 days. Results are reported as the mean $\pm \mathrm{SD}$ (see Materials and methods). ${ }^{*} \mathrm{P}<0.05$.
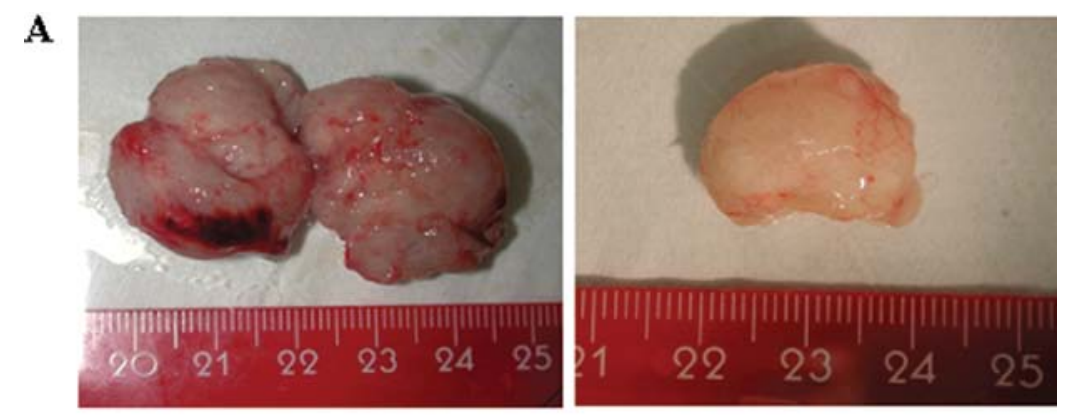

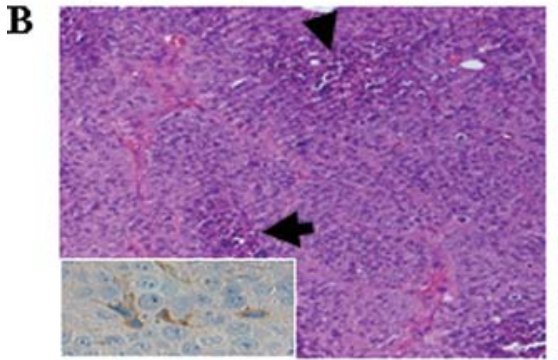

Control

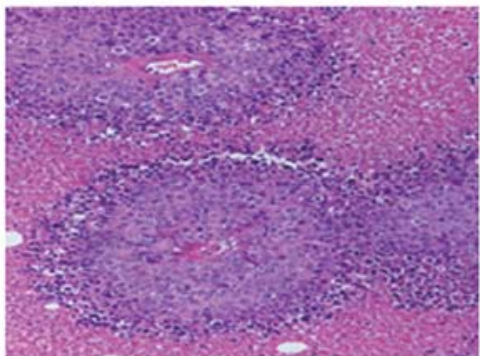

Sunitinib $(40 \mathrm{mg} / \mathrm{kg} / \mathrm{day})$

Figure 2. Tumor masses and histological examination of xenograft tumors. (A) Xenograft tumors after 11 days of treatment with vehicle (left) (control) or sunitinib $40 \mathrm{mg} / \mathrm{kg} / \mathrm{day}$ (right). (B) On the left, hematoxylin and eosin stain (H\&E, x200) of one non-treated (control) tumor: neoplastic cells grow in solid areas with well developed small venules and capillaries, the latter highlighted in the inset by CD31 immunohistochemistry (x600); only microfoci of tumor necrosis are present in the picture (arrows) and due to the developed microvasculature there is no peritheliomatous arrangement of tumor cells around a feeding vessel; on the right, $\mathrm{H} \& \mathrm{E}$ stain (x200) of one sunitinib-treated tumor xenograft: due to the lack of small venules and capillaries, neoplastic cells form peritheliomatous structures that have been spared from necrosis due to their proximity to a centrally located feeding vessel; note the large amounts of necrotic tumor tissue that stains with eosin.

whole tumor sections were compared by the unpaired t-test. The relationship between tumor necrosis and MVD was analyzed by the Pearson correlation test. All statistical analyses were carried out using GraphPad Prism software (GraphPad, San Diego, CA, USA).

\section{Results}

Effects of sunitinib on the growth of xenograft tumors. Antitumor activity of sunitinib in vivo was investigated in a renal tumor xenograft mouse model. Administration of sunitinib (40 mg/kg/day) or vehicle was initiated after HEK293 cell implantation on xenografts, when the average tumor volume was approximately $500 \mathrm{~mm}^{3}$. Treatment with sunitinib resulted in a significant inhibition of tumor growth ( $\sim$-fold after 11 days treatment) (Fig. 1A). A significant reduction of the weight of the collected tumoral masses was also observed in tumor-bearing mice treated with sunitinib compared to untreated tumors (Fig. 1B).

Effects of sunitinib on microvessel density and histological analysis of tumor necrosis. Reduction of the the mass associated to macroscopic reduction of vascularization was evident at the end of the treatment (Fig. 2A). Histologic 
A

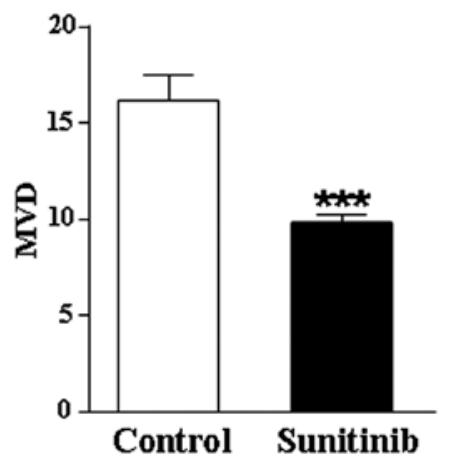

B

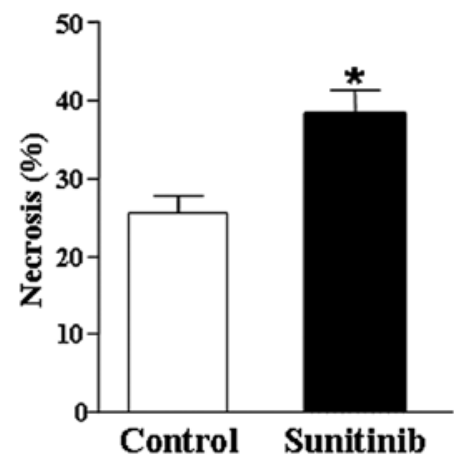

$\mathrm{C}$

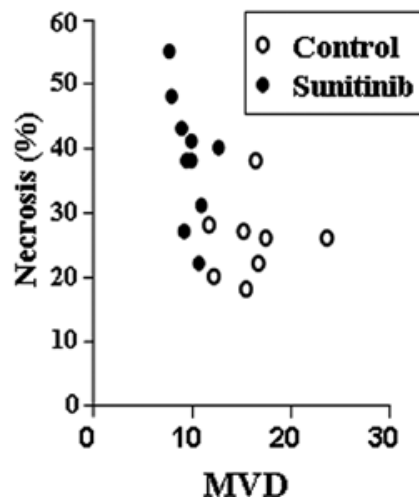

Figure 3. Evaluation of microvessels density (MVD) and necrosis. (A) MVD values and (B) percentage of tumor necrosis for non-treated control tumors (eight whole tumor sections from two tumors) vs. sunitinib-treated tumors (ten whole tumor sections from three tumors). White bars, non-treated (control) tumor xenografts; black bars, treated tumor (sunitinib) xenografts; all bars represent mean values and standard error of the mean. Statistical analysis was performed by unpaired t-test. "P $<0.05 ;{ }^{* * * *} \mathrm{P}<0.001$. (C) MVD and the percentage of tumor necrosis were evaluated on each of eight whole tumor sections from two nontreated control tumors and each of ten whole tumor sections from three sunitinib-treated tumors. Sunitinib-treated tumors have lower MVD values and a higher proportion of necrosis compared with controls. Necrosis is inversely and significantly correlated to MVD (Pearson correlation coefficient $\mathrm{r}=-0.55$, $\mathrm{P}=0.0174)$.

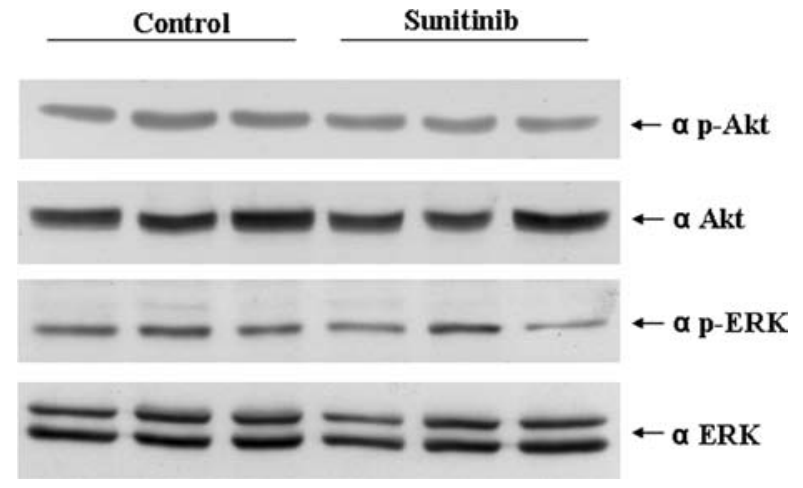

Figure 4. Effects of sunitinib on ERK and Akt phosphorylation. Immunoblot analysis of ERK, phospho-ERK, Akt and phospho-Akt expression of in vivo tumors treated with sunitinib for 11 days. One experiment, performed with protein extracts from three tumors of each group (treated and untreated) representative of three different experiments is shown (see Materials and methods).

examination of tumor sections showed marked differences between non-sunitinib-treated control tumor xenografts and sunitinib-treated tumors. In the control tumors, neoplastic cells formed solid areas up to 3-4 $\mathrm{mm}$ in diameter with sparse microfoci of necrosis. Small venules and capillaries formed a well developed network within the solid areas of growth (Fig. 2B, left panel). Moreover, in control xenografts, tumor necrosis also formed irregularly distributed patches (not shown).

In contrast, in sunitinib-treated tumor xenografts, small venules and capillaries were poorly developed, sometimes virtually absent. As a result, neoplastic cells survived only in the proximity of a centrally located feeding vessel, resulting in characteristic peritheliomatous structures uniformly distributed throughout the tumor, separated by large confluent areas of tumor necrosis (Fig. 2B, right panel). Overall microvessel density was higher in controls than in sunitinib-treated tumor xenografts $(\mathrm{P}=0.0001)$ (Fig. 3A), while the amount of tumor necrosis was smaller (Fig. 3B; $\mathrm{P}=0.0056$ ). Measurements of average microvessel density and percentage of tumor necrosis in the same whole tissue section from both control and sunitinibtreated tumor xenografts show that necrosis is inversely and significantly correlated to microvessel density (Fig. 3C; Pearson correlation coefficient $r=-0.55, P=0.0174)$. No signs of epithelial-mesenchimal transition were observed (Fig. 2).

Effects of sunitinib on growth regulatory signal transduction pathways. The activity of some signal transduction pathways involved in the cell growth regulation was investigated. Our attention was focused on RAF/MEK/ERK and PI3K/PTEN/ Akt-dependent pathways. The levels of phospho-Akt, phospho-ERK and their respective non-phosphorylated proteins were evaluated by Western blot analysis in protein extracts from the tumor masses of three animals of each group of sunitinib-treated and untreated mice. As shown in Fig. 4, no significant differences in either p-Akt or p-ERK levels were observed.

Effects of sunitinib on the expression of angiogenic factors. Finally, we examined the effects of sunitinib on the proangiogenic VEGF-A and VEGFR1,2 and 3 gene expression (Fig. 5). No significant changes in VEGF-A and VEGFR1, 2 and 3 mRNA levels resulted in xenograft tumors treated with sunitinib compared to controls.

\section{Discussion}

Sunitinib is a multikinase inhibitor currently used for the treatment of some solid malignancies, including advanced RCC and GIST after disease progression or intolerance to imatinib mesylate therapy. Moreover, objective partial responses were reported in several studies enrolling patients with thyroid cancer, neuroendocrine cancer, sarcoma, non-small cell lung cancer and melanoma, supporting the basis for phase II/III clinical development program of this drug (12). Preclinical studies have clearly demonstrated that sunitinib inhibits the VEGF, stem cell factors and PDGF-dependent 

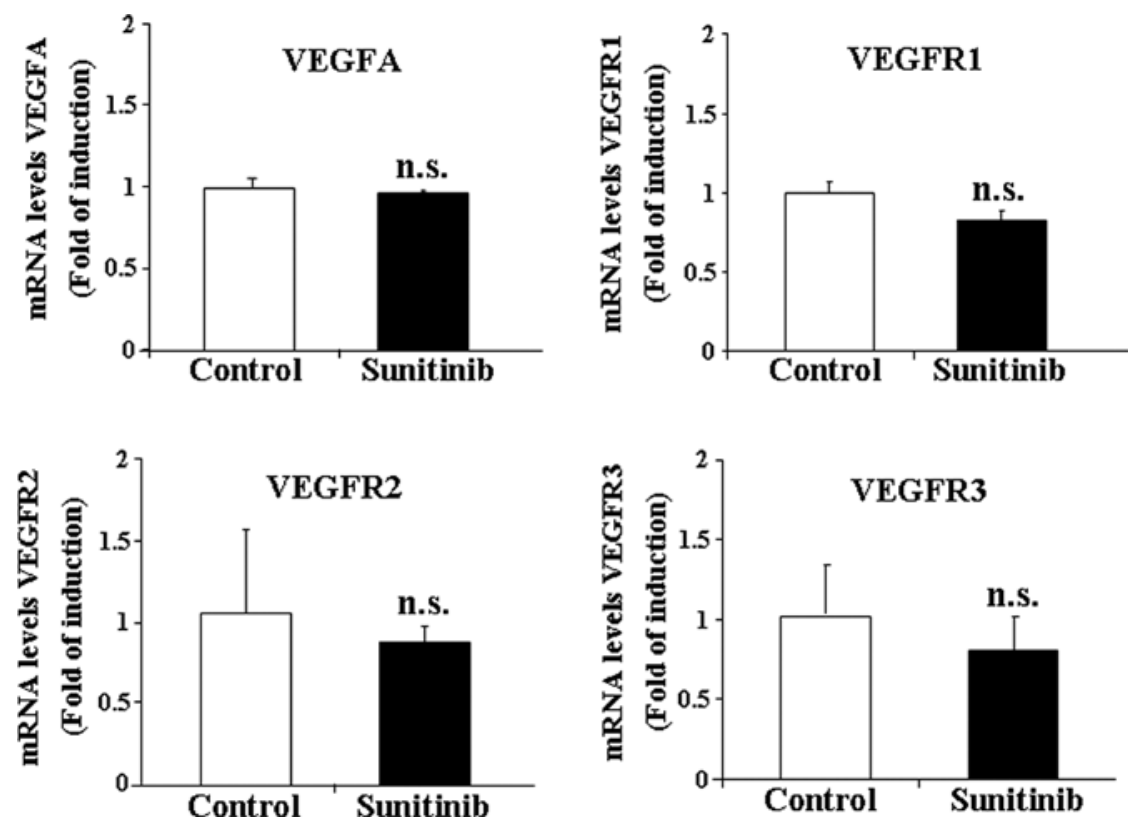

Figure 5. Expression of VEGFA and VEGFR1, 2 and 3 mRNA levels. Total-RNA was extracted from control and treated (sunitinib $40 \mathrm{mg} / \mathrm{kg} / \mathrm{day}$ ) mice after their sacrifice at the end of the experiments and the transcript levels of VEGF and VEGFR1, 2 and 3 genes analyzed by quantitative RT-PCR, as described in Materials and methods. Data are expressed as the mean \pm SD of values obtained from at least three different experiments. Statistic analysis was performed using the t-test. n.s., $P>0.05$.

proliferation and migration of human endothelial cells, and, in xenograft models from numerous tumor types, inhibited cancer angio-genesis decreasing tumor microvessel density (12). Resistance did not appear in these models, suggesting that the anti-angiogenic action mediated by the block of phosphorylation of VEGF-R and PDGF-R is effective against well-established tumor types. In contrast, a considerable group of patients with RCC or GIST do not respond to this drug or eventually develop resistance to sunitinib therapy $(6,7)$. At present the molecular mechanism underlying such a resistance are not fully clarified. Two main theories have been proposed to account for acquired resistance. The first one suggests the occurrence of an 'angio-genic escape' substained by up-regulation of associated proangiogenic growth factors. The second supports the role of alternative kinase pathways, a mechanism proposed as responsible for the resistance to kinase inhibitors developing in many tumor cells $(8,9)$. In particular, in GIST, cKIT and PDGFR mutations have been described (13), suggesting that overcoming resistance in these tumors will require combinatorial approaches that target other characteristics of the tumors. The same strategy is suggested for the non-responsive RCC, in which therapies with mTOR inhibitors are currently recommended after VEGF-targeted treatment (14). In these tumors, the presence of acquired mutations is not thought to be involved in the resistance $(15,16)$. Thus, a complete understanding of sunitinib action against tumor cells is crucial to identify an ideal combination with other targeted agents able to challenge the mechanism of resistance.

In this study, we have tested the effects of sunitinib on other tumor cell TK-mediated growth regulatory pathways in HEK293-induced xenograft tumors. In accordance with data obtained in various other models, treatment with sunitinib $40 \mathrm{mg} / \mathrm{kg} /$ day determined a tumor growth inhibition, clearly caused by reduced vascularization. The microvessel density, in fact, was greatly reduced in the sunitinib treated animals and such effect paralleled the presence of necrotic tumor cells. In accordance with responsiveness to the treatment, no signs of epithelial-mesenchimal transition, proposed as markers of resistance to sunitinib treatment in RCC (17), were observed. However, in the tumor tissue, both ERK and Akt phosphorylation was not affected, suggesting that the correspondent pathways, known for their role as growth-promoting and anti-apoptotic (18), resulted still activated in the surviving tumor cells irrespective of the treatment with sunitinib. Such findings, therefore, suggest that these pathways may play a role for the selection of cell clones which may determine, in long-term, secondary resistance to the treatment. Thus, agents targeting such kinases or their downstream pathways may represent valid candidates for a combined treatment with sunitinib in resistant tumors. Indeed, mTOR inhibitors have been tested in sunitinib resistant RCC, although, when used as single agent, a compensatory up-regulation of PI3 kinase has been reported (19). Development of novel specific combination of targeted agents are therefore eagerly awaited, taking into consideration the preference for those agents with the minimal toxic effects.

Another important issue in the treatment with the novel anticancer targeted agents, and in particular for sunitinib, is represented by the need of biomarkers able to predict the individual response to the treatment. In patients with objective response to sunitinib, an early increase in VEGF serum levels with a decrease of VEGFR-2 (in RCC) or VEGFR-2 and 3 levels (in GIST) has been reported and interpreted as the result of hypo-oxigenation of the tumor mass $(7,3)$. Their assessment in patients subjected to sunitinib treatment has been therefore proposed as biomarkers of its efficacy. In the xenograft tumors treated with sunitinib, examination of VEGF and its receptor mRNA expression levels at the end of 
the treatment, did not show any significant differences versus non-treated tumors. Probably, in our experimental model, other factors occurred counteracting the effects of hypoxia, or the dislocation of the surviving tumor cells around the remaining vessels (as clearly shown in Fig. 2B) defended the cells from the damage induced by reduced nutrition and its secondary effects. The development of new biomarkers, therefore, remains a critical goal for the optimization of any kind of treatment (20).

In conclusion our findings indicate that sunitinib, by acting primarily through an anti-angiogenic mechanism, does not affect some important molecular growth regulatory pathways of cancer cells. Therefore, only a combination with other selective TK inhibitors may offer valid opportunities to overcome sunitinib resistance.

\section{Acknowledgements}

This study was supported by the 'Fondazione Umberto Di Mario ONLUS' and from the 'Fondazione Cassa di Risparmio di Perugia'.

\section{References}

1. Abrams TJ, Murray LJ, Pesenti E, et al: Preclinical evaluation of the tyrosine kinase inhibitor SU11248 as a single agent and in combination with 'standard of care' therapeutic agents for the treatment of breast cancer. Mol Cancer Ther 10: 1011-1021, 2003.

2. Mendel DB, Laird AD, Xin X, et al: In vivo antitumor activity of SU11248, a novel tyrosine kinase inhibitor targeting vascular endothelial growth factor and platelet-derived growth factor receptors: determination of a pharmacokinetic/pharmacodynamic relationship. Clin Cancer Res 1: 327-337, 2003.

3. Le Tourneau C, Raymond E and Faivre S: Sunitinib: a novel tyrosine kinase inhibitor. A brief review of its therapeutic potential in the treatment of renal carcinoma and gastrointestinal stromal tumors (GIST). Ther Clin Risk Manag 2: 341-348, 2007.

4. Patyna S, Laird AD, Mendel DB, et al: SU14813: a novel multiple receptor tyrosine kinase inhibitor with potent antiangiogenic and antitumor activity. Mol Cancer Ther 7: 1774-1782, 2006.

5. Motzer RJ, Hutson TE, Tomczak P, et al: Overall survival and updated results for sunitinib compared with interferon alfa in patients with metastatic renal cell carcinoma. J Clin Oncol 27: 3584-3590, 2009.
6. Rini BI and Atkins MB: Resistance to targeted therapy in renalcell carcinoma. Lancet Oncol 10: 992-1000, 2009.

7. Gounder MM and Maki RG: Molecular basis for primary and secondary tyrosine kinase inhibitor resistance in gastrointestinal stromal tumor. Cancer Chemoter Pharmacol 67 (Suppl 1): S25-S43, 2011.

8. Zhang J, Yang PL and Gray NS: Targeting cancer with small molecule kinase inhibitors. Nat Rev Cancer 9: 28-39, 2009.

9. Stommel JM, Kimmelman AC, Ying H, et al: Coactivation of receptor tyrosine kinases affects the response of tumor cells to targeted therapies. Science 318: 287-290, 2007.

10. Sponziello ML, Bruno R, Durante C, et al: Growth factor receptors gene expression and Akt phosphorylation in benign human thyroid nodules are unaffected by chronic thyrotropin suppression. Horm Metab Res 43: 22-25, 2011.

11. Weidner N: Intratumor microvessel density as a prognostic factor in cancer. Am J Pathol 147: 9-19, 1995.

12. Mena CA, Pulido EG and Guillen-Ponce C: Understanding the molecular-based mechanism of action of the thyrosine kinase inhibitor: sunitinib. Anticancer Drugs 21 (Suppl 1): S3-S11, 2010.

13. Wang WL, Conley A, Reynoso D, Nolden L, Lazar AJ, George S and Trent JC: Mechanisms of resistance to imatinib and sunitinib in gastrointestinal stromal tumor. Cancer Chemoter Pharmacol 67 (Suppl 1): S15-S24, 2011.

14. Powles T, Chowdhury S, Jones R, et al: Sunitinib and other targeted therapies for renal cell carcinoma. Br J Cancer 104: 741-745, 2011.

15. Valent P: Emerging stem cell concepts for imatinib-resistant chronic myeloid leukaemia: implications for the biology, management, and therapy of the disease. Br J Haematol 142: 361-378, 2008.

16. Rini BI, Wilding G, Hudes G, et al: Phase II study of axitinib in sorafenib-refractory metastatic renal cell carcinoma. J Clin Oncol 27: 4462-4468, 2009.

17. Hammers HJ, Verheul HM, Salumbides B, et al: Reversible epithelial to mesenchymal transition and acquired resistance to sunitinib in patients with renal cell carcinoma: evidence from a xenograft study. Mol Cancer Ther 6: 1525-1535, 2010.

18. McCubrey JA, Steelman LS, Abrams SL, et al: Roles of the RAF/MEK/ERK and PI3K/PTEN/AKT pathways in malignant transformation and drug resistance. Adv Enzyme Regul 46: 249-279, 2006.

19. O'Reilly KE, Rojo F, She QB, et al: mTOR inhibition induces upstream receptor tyrosine kinase signaling and activates Akt. Cancer Res 66: 1500-1508, 2006.

20. Faivre S, Demetri G, Sargent W and Raymond E: Molecular basis for sunitinib efficacy and future clinical development. Nat Rev Drug Discov 6: 734-745, 2007. 